\title{
Searching for Wide Binary Brown Dwarfs around Nearby Stars
}

\author{
Loïc Albert, René Doyon, and Daniel Nadeau \\ Université de Montréal, Département de physique, C.P. 6128 Succ. \\ Centre-ville, Montréal, H3C 3J7, Québec, Canada
}

\begin{abstract}
We have started a program to search for Brown Dwarf (BD) companions to nearby stars $(5 \leq d \leq 10 \mathrm{pc}$ ) on long period orbits (up to $2000 \mathrm{AU}$ ) using wide field optical/infrared imaging. Our goals in surveying a volume-limited sample of stars are: 1 ) to determine the binary fraction of widely separated BDs and help constrain the BD formation processes, 2) to search for very cold BDs, 3) to provide a better determination of the age and luminosity of a BD sample. Observations are currently conducted at three observatories, CFHT $(3.6 \mathrm{~m})$, CTIO $(4 \mathrm{~m})$ and Observatoire du mont Mégantic $(1.6 \mathrm{~m})$ and should yield a sample of $\sim 100$ star systems to a detection limit of $J \approx 20$.
\end{abstract}

\section{Motivation, Detection Technique and Current Status}

Radial velocity studies (Marcy \& Butler, 2000) and high-resolution imaging studies (Oppenheimer et al., 2001) have revealed that less than $~ 1 \%$ of F-M main sequence stars have a BD companion at small separation ( $\leq 100$ A.U.). This has been coined as the "Brown Dwarf Desert", in contrast with the $\sim 15 \%$ binary fraction of stellar companions around $\mathrm{G}$ type stars in the same distance interval (Duquennoy \& Mayor, 1991). But is the BD desert really dry at larger orbital distances? An analysis by Gizis et al. (2001) showed that a few 2MASS BDs were actually associated to nearby stars in very wide orbits ( 1000 A.U.). About 11 BDs are currently known to orbit around main sequence stars. Eight of them would escape current high-resolution searches which cover orbits no wider than 250 A.U. Only one published study was specifically designed to look for high-angular separation BDs (Simons et al., 1996, and Hinz et al., 2002). They found no BD out of 66 surveyed stars, but their search was limited to $J=16.5$.

Our BD detection technique is described in Figure 1. It is most appropriate for discovering $\mathrm{T}$ type BDs which have $J-H=0.0 \pm 0.3$. Our candidates will require further spectroscopic confirmation as well as new direct imaging observations to study their proper motion and confirm their physical association to the primary star. To date, 70 stellar systems have been surveyed in the $I, J$ and $H$ bands and we have started data reduction. The current number of known widely separated BDs suggests we may find a BD companion around $5-15 \%$ of our targets. 


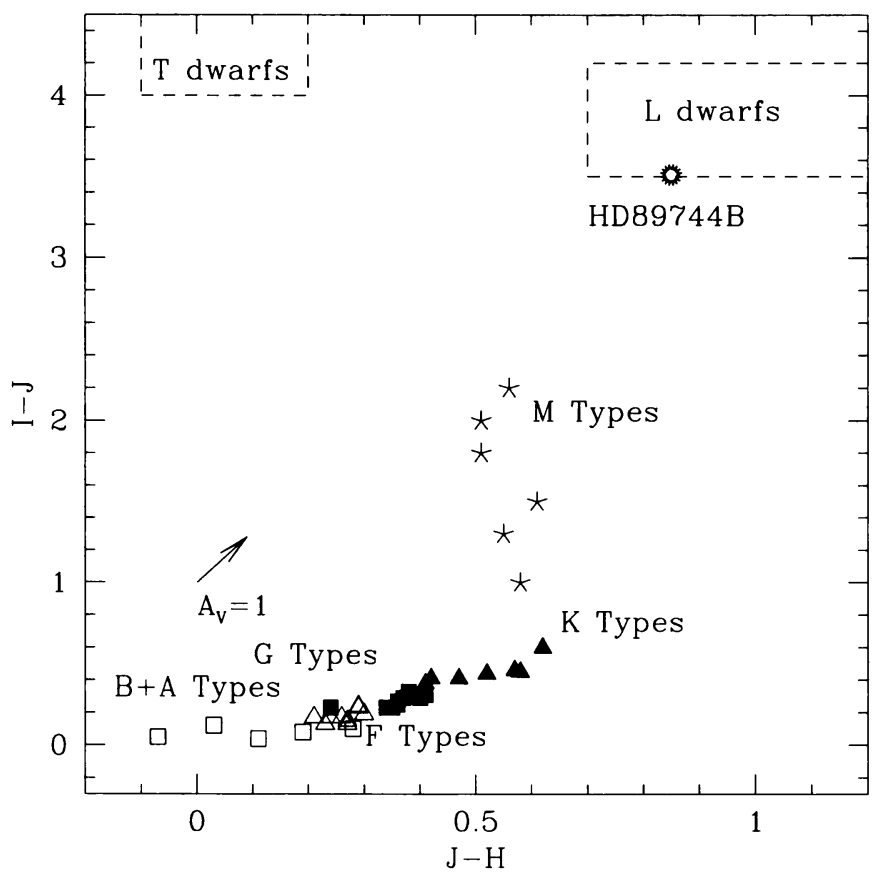

Figure 1. $I-J$ vs. $J-H$ color-color plot used to isolate BD candidates among field point sources. Most $\mathrm{T}$ type BDs have $J-H$ colors of $0.0 \pm 0.3$ like $\mathrm{B}, \mathrm{A}$ and $\mathrm{G}$ type stars. The degeneracy is lifted with the $I$ band, T BDs have $I-J \geq 4-5$, i.e. T BDs should not appear in the $I$ image. With similar quality images in $I, J$ and $H$, good $J$ and $H$ photometry ( $10 \sigma$ detection, $\pm 0.1 \mathrm{mag}$ precision) is achieved while detecting objects $1.5 \mathrm{mag}$ fainter in $I(3 \sigma)$. As for L type BDs, they are more difficult to distinguish from very late $M$ stars, and within 10 pc most should already have been discovered by 2MASS and DENIS, so they are not our primary objective in this survey.

\section{References}

Duquennoy, A., Mayor, M., 1991, A\&A, 248, 485

Hinz, J. L., McCarthy, D. W., Simons, D. A., Henry, T. J., Kirkpatrick, J. D., McGuire, P. C., 2002, AJ, 123, 2027

Gizis, J. E., Kirkpatrick, J. D., Burgasser, A., Reid, I. N., Monet, D. G., Liebert, J., Wilson, J. C., 2001, ApJL, 551, 163

Kirkpatrick, J. D., 2002, these proceedings

Marcy, G. W., Butler, R. P., 2000, PASP, 112, 137

Oppenheimer, B.R., Golimowski, D. A., Kulkarni, S. R., Matthews, K., Nakajima, T., Creech-Eakman, M., Durrance, S. T., 2001, AJ, 121, 2189

Simons, D. A., Henry, T. J., Kirkpatrick, J. D., 1996, AJ, 112, 2238 